\title{
A Study of English-Indonesian Neural Machine Translation with Attention (Seq2Seq, ConvSeq2Seq, RNN, and MHA)
}

\author{
A Comparative Study of NMT on English-Indonesian \\ Diyah Puspitaningrum \\ Computer Science Department, University of Bengkulu, Bengkulu, Indonesia \\ diyahpuspitaningrum@gmail.com
}

\begin{abstract}
In recent years, Neural Machine Translation (NMT) with attention mechanisms has emerged in research and industry. This study discusses the essentials of NMT (Seq2Seq, Convolutional Seq2Seq (ConvSeq2Seq), Recurrent Neural Networks (RNN), and Multi-Head Attention (MHA)) while implemented in formal passages in EnglishIndonesian and Indonesian-English. The experimental results for ConvSeq2Seq achieve up to 38.99 BLEU sentence scores, 43.23 BLEU corpus scores, and 39.48 GLEU corpus scores over the Seq2Seq English-Indonesian. For Indonesian-English, the results for ConvSeq2Seq achieved as follows: up to 42.59 BLEU sentence scores, 42.91 BLEU corpus scores, 41.05 GLEU corpus scores, and 1356.65 WER scores over RNN and MHA. Thus, while ConvSeq2Seq tends to be the supremacy, this literature also describes the combination of architectures and specific fine-tuning strategies as a discussion.
\end{abstract}

\section{KEYWORDS}

Seq2Seq, RNN, convolutional Seq2Seq, multi-head attention

\section{ACM Reference Format:}

Diyah Puspitaningrum. 2021. A Study of English-Indonesian Neural Machine Translation with Attention (Seq2Seq, ConvSeq2Seq, RNN, and MHA): A Comparative Study of NMT on English-Indonesian. In 6th International Conference on Sustainable Information Engineering and Technology 2021 (SIET '21), September 13, 14, 2021, Malang, Indonesia. ACM, New York, NY, USA, 10 pages. https://doi.org/10.1145/3479645.3479703

\section{INTRODUCTION}

NMT is an extensive neural network trained to translate a source sequence of one language into a target sequence of another language. NMT consists of two main components: encoder and decoder. The encoder computes a representation of each source sentence; the decoder then generates translation one word at each time and hence decomposes the conditional probability of translating a source $(\mathrm{x} 1, \mathrm{x} 2 \ldots \mathrm{xn})$ sentence into a target sentence (y1, y2 ...yn). NMT with attention mechanisms has emerged in research and industry because NMT requires minimal domain knowledge and is conceptually very simple. NMT can generalize well to very longword sentences. NMT has achieved state-of-the-art performances in large-scale translation tasks of many languages globally, such as English to French or English to German, as a well-known example.

ACM acknowledges that this contribution was authored or co-authored by an employee contractor or affiliate of a national government. As such, the Government retains a nonexclusive, royalty-free right to publish or reproduce this article, or to allow others to do so, for Government purposes only.

SIET '21, September 13, 14, 2021, Malang, Indonesia

(C) 2021 Association for Computing Machinery.

ACM ISBN 978-1-4503-8407-0/21/09..\$15.00

https://doi.org/10.1145/3479645.3479703
From the perspective of memory usage, NMT has the advantage of not storing the massive size of phase tables and language models. We have investigated many works of literature in NMT [34], [20], [26], [7], [8]. This study focuses on the essentials of NMT (Seq2Seq, ConvSeq2Seq, RNN, and MHA) while implemented in formal passages in English-Indonesian and Indonesian-English. We also explain several fine-tuning strategies to improve NMT systems.

We organize the remaining paper as follows. Section 2 provides background on the essentials of four NMT approaches used in the experiment. Section 3 is the experimental setup of NMT implementation in Indonesian to English and English to Indonesian. Section 4 contains results, the fine-tuning strategies, and a discussion of a proposal for future improvement of the existing NMT methods. Finally, in Section 5 we draw conclusions.

\section{NMT APPROACHES}

The next subsections provide instructions on how to insert figures, tables, and equations in your document.

\subsection{Seq2Seq}

Sequence-to-sequence (Seq2Seq) [28], [5] is a deep learning model that takes a sequence of items, such as words, letters, time series, and outputs another sequence of items. The seq2Seq approach has achieved much success in machine translation, text summarization, and image captioning. An example of a sequence-to-sequence task is the translation: the input is a sequence of words, and the output is the translated sequences of the words. Figure 1 shows a workflow scheme of Seq2Seq with attention.

The basics of Seq2Seq are as follows. The model is composed of an encoder-decoder. The encoder gets the context of the input sequence in a hidden state vector and sends it to the decoder, producing the output sequence. Since the task is sequence-based, both encoder and decoder tend to use some form of, for example, RNNs, LSTMs, GRUs. The size of the hidden state vector can be of any dimension, usually a power of 2 and a large number $(256,512$, 1024), representing the complexity of the complete sequence and the domain.

The attention mechanism decides which source parts are more critical than others at every decoder step. When a NMT passes a source sentence in a language to an encoder, the encoder encodes the complete information of the input sequence into a single vector called a context vector. The system then passes the context vector into the decoder. The decoder produces a translated output sequence in the target language. The attention mechanism gives representations for all source tokens, not only the last states. The mechanism lets a model focus on different input parts by scoring 


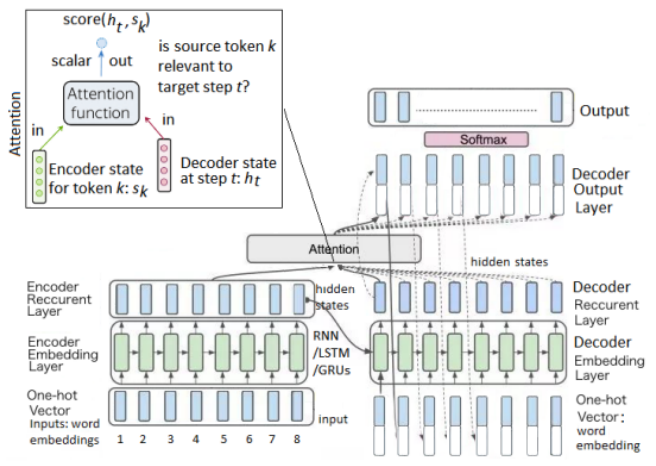

Figure 1: Architecture of Seq2Seq with attention.

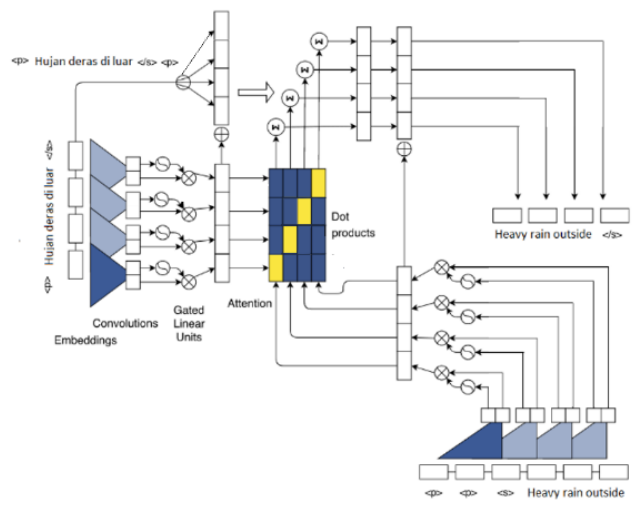

Figure 2: ConvSeq2Seq with attention, implemented in Indonesian-English.

how relevant source token $\mathrm{k}$ for target step $\mathrm{t}$ uses the softmax attention function. Other popular techniques to do attention are dot-product, bilinear function (or "Luong attention," [18]), and multilayer perceptron (or "Bahdanau attention," [2]). See [28] for original work of Seq2Seq.

\subsection{ConvSeq2Seq}

In machine translation, the Seq2Seq approach using CNN has showed to outperform the traditional phrase-based models significantly [23], [36], [33]. CNN themselves are less common for sequence modeling, despite several advantages [31], [15]. First, the convolutions create representations for fixed-size contexts. This situation is different from RNNs, which learned one input at a time in sequence. In contrast, in convolution neural networks, if we are stacking several layers on top of each other, this will create a larger adequate context size of the network. By doing this, we allow convolution neural networks to precisely control the maximum length of dependencies to be modeled. $\mathrm{CNN}$ do not depend on the computations of the previous time step and therefore allow parallelization over every element in a sequence.

Figure 2 shows the ConvSeq2Seq approach for the NMT task. Computations over all elements can be completely parallel during training. Optimization is easier since the number of nonlinearities is fixed and independent of the input length. [10] introduce Gated
Linear Units instead of Gated Recurrent Units which eases gradient propagation and equips each decoder layer the separate/particular attention (multi-hop attention). [10] outperform in both WMT'14 English-German and English-French translation tasks of [33]. Below are the essentials of ConvSeq2Seq for the NMT task (further see [10] for original work of ConvSeq2Seq):

- Position embedding. The input element representations for Indonesian-English "Hujan deras di luar" (Figure 2) fed into an encoder combine word and position embedding of input elements. The output elements generated by the decoder network proceed similarly to results output element representations that are then is being fed back into the decoder network. Position embeddings are helpful in this architecture because the position embeddings give the model a sense of which portion of the sequence in the input or output it is currently deal.

- Convolutional block structure. Both encoder and decoder networks share a simple block structure that computes intermediate states based on a fixed number of input elements.

- Multi-step attention. ConvSeq2Seq introduces a separate attention mechanism for each decoder layer. To compute the attention, we combine the current decoder state with an embedding of the previous target element. ConvSeq2Seq computes the decoder layer, the attention, and there is a dot-product between the decoder state summary and every output of the last encoder block. The conditional input for the current decoder layer is a weighted sum of the encoder outputs and the input elements embedding. For example, in Figure 2, the decoder output for the Indonesian-English task is: "Heavy rain outside."

\subsection{RNN}

RNN has achieved state-of-the-art performance in neural machine translation. The first successful RNN based NMT is in [28]. They used a deep RNN model and got a performance that approximates the best result of statistical machine translation. Furthermore, the attention mechanism significantly improves the translation performance of the machine translation model. One example of RNN based machine translation is Google Translation. Furthermore, [36] have designed Fast-Forward Connections for RNN (LSTM), allowing a deeper network implementation and better performance.

RNN can be viewed as a loop or copy-pasting action of the same network repeatedly, with every new copy-paste action affects adding a bit more information than the preceding. The applications for the RNN are vastly different from traditional neural networks because they do not have the output and input set as a concrete value. As the input or output, the RNNs take sequences. It is a sequence-tosequence model. It takes a sequence input from a source language and outputs a sequence in a target language. Figure 3 shows the implementation of RNN as follows: "Providing technical guidance and evaluation on improvements to the legal system" (English) to "Memberikan panduan teknis dan evaluasi pada sistem hukum" (Indonesian).

Given a hidden state $\left(\mathrm{h}_{\mathrm{i}}\right)$ that encodes all the information in the sentence of the target language, at each time step, an RNN picks only a single word $\left(\mathrm{w}_{\mathrm{i}}\right)$. The $\mathrm{RNN}$ allows varying input and 


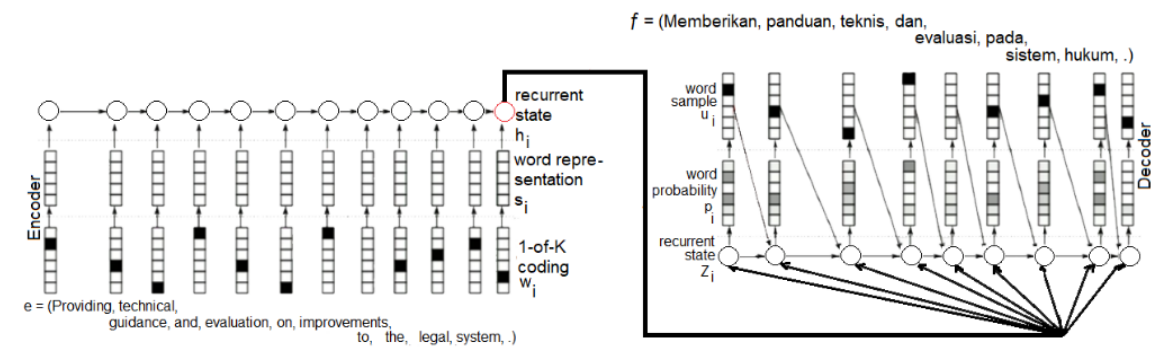

Figure 3: RNN with attention, implemented in Indonesian-English.

output length. It updates its hidden state $\left(\mathrm{h}_{\mathrm{i}}\right)$ in a deterministic nonlinear way and then sends the chosen word back to the network as input. The attention mechanism works as follows. First, using the fully connected network, RNN computes the recurrent state $\mathrm{Z}_{\mathrm{i}}$ based on the summary vector $\left(\mathrm{h}_{\mathrm{i}}\right)$ of the original sentence, the preceding word sample $\left(\mathrm{u}_{\mathrm{i}-1}\right)$, and the preceding recurrent state $\left(\mathrm{z}_{\mathrm{i}-1}\right)$. Next, we score each target word based on the likely degree to follow all the preceding translated words given the source sentence. Next, RNN scores each word and then computes their probabilities using softmax normalization. The resulting context vectors enable the decoder to focus on certain input parts when predicting its output. Finally, RNN selects a word after obtaining probabilities over the target words by sampling the distribution to produce an output. After reaching the last word, RNN iteratively computes the hi, scoring, and softmax normalizing until RNN reaches the sentence's end. See [28] for further RNN reading.

RNNs are very powerful because the distributed hidden state allows RNN to store information about the past efficiently. Nonlinear dynamics allow them to update their hidden state in complicated ways. RNN is purely deterministic and weight sharing. The limitation of RNN is on the vanishing gradient problem. The vanishing gradient problem is the problem that occurs in any network that uses gradient-based optimization techniques. For example, when calculating the gradients of loss for the weights in backpropagation, the gradients get very small as the backpropagation algorithm moves through the network. The situation causes the earlier layers to learn way slower than later ones. It then reduces the effectiveness of RNNs as they often cannot fully consider long sequences. As the gap between the needed information becomes prominent, an RNN gets less and less effective. A standard solution to this problem is to use activation functions that do not cause vanishing gradients, such as RELU function, instead of other existing activation functions such as a sigmoid function or hyperbolic tangent. An alternative and a better solution are using Long-Short Term Memory Networks (LSTM). The LSTM architecture consists of memory blocks (recurrently connected subnets). The memory blocks are like the memory chips in a digital computer. Each block contains more self-connected memory cells and three multiplicative units that provide continuous analogs of the cells' write, read and reset operations [27].

\subsection{Multi-head Attention}

MHA uses stacked multi-head self-attention and fully connected layers within the encoder-decoder framework instead of a single attention function. First, MHA linearly projects the queries, keys, and values $\mathrm{h}$ times with different, learned linear projections to $D_{Q}$, $D_{k}$, and $D_{V}$ model dimensions in MHA, respectively (Figure 2 in [30]). These logically split Query (or $Q$ ), Key (or $K$ ), and Value (or $V)$ are as one input for each attention head (Figure 4 (right)). Then, the MHA performs an attention function parallel to these projected queries, keys, and values. After that, they are concatenated and projected, resulting in $d$-dimensional output values. Thus, attention heads share the same Linear layer that operates on the data matrix in their logical section.

Figure 4 (left) illustrates all types of attention in the MHA model. The implementation of attention can be self-attention or global attention, for example, 1) In self-attention in the encoder, where the input sequence pays attention to itself; 2) In self-attention in the decoder, where the target sequence pays attention to itself; 3 ) In Encoder-Decoder-attention in the decoder, the target sequence pays attention to the input sequence. MHA, in this case, allows the model to jointly attend to information from different representation subspaces at other positions. Figure 4 (right) illustrates the MHA steps in [30] shortly. After feed the input sentence $X$ (Step 1) and then do embedding on each word of $X$ (Step 2), the next step is splitting the chunk words into the $V, K$, and $Q$ (Step 3). And then transform those divided $V / K / Q$ using densely linear connected layers. After that, at Step 4, MHA computes attention weights, reweight the $V$, and takes the summation of the reweighted $V$. The last step, at Step 5, is concatenating (or stacking) the resulting summations and pass through the result of Step 4 through other connected layers. For example, if we have a fixed number of heads is eight. At the same time, the sentence input consists of 11 tokens, and the dimension of the embedding vector $=512$ means that to express the sentence, MHA splits every token into 512 divides by 8 , resulting in 64 dimensional eight vectors of word chunks (11x64 matrices). All processes then are calculated in those eight heads of multi-head attention. For example, if the model has six layers, the encoder has $6 \times 8=48$ heads; thus, the encoder studies the relations of input tokens on 48 standards.

MHA attention process. The process concatenates the resulting $\mathrm{Z}$ matrices and multiplying them with weight matrix $\mathrm{W}^{\mathrm{O}}$ to produce the layer's output according to [30] as follows:

$$
\operatorname{Attention}(Q, K, V)=\operatorname{softmax}\left(\frac{Q K^{T}}{\sqrt{d_{k}}}\right) V
$$

$$
\text { multihead }(Q ; K ; V)=\text { Concat }\left(\text { head }_{1}, \ldots, \text { head }_{h}\right) W^{O}
$$



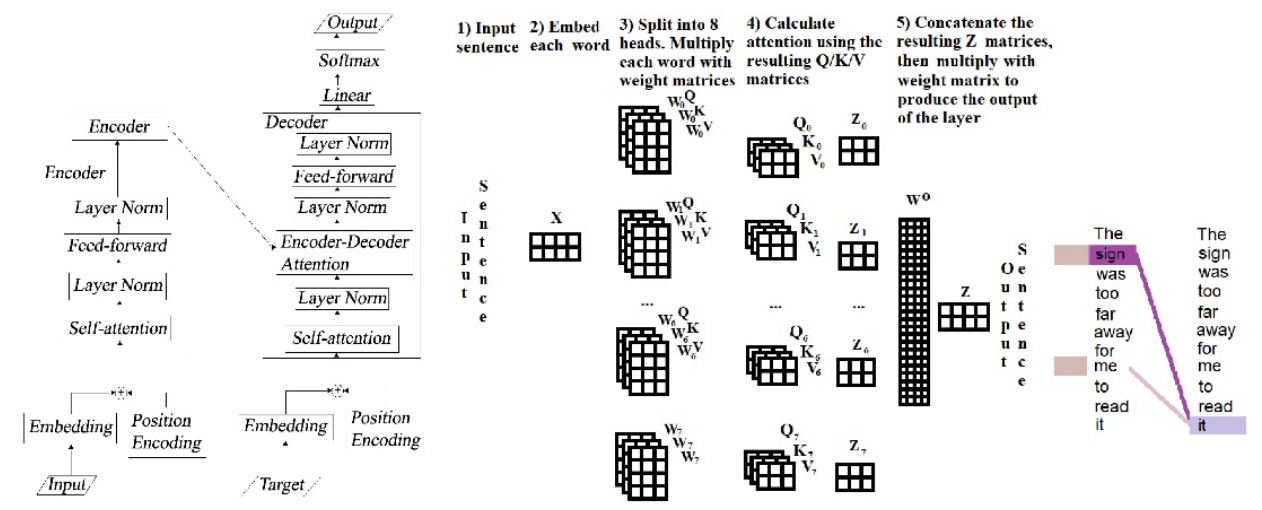

Figure 4: Multi-head with an attention mechanism. MHA model (Left). MHA steps (Q/K/V) (Right).

$$
\operatorname{head}_{i}=\operatorname{Attention}\left(Q W_{i}^{Q}, K W_{i}^{K}, V W_{i}^{V}\right)
$$

For example, in Figure 4 (right), given such a sentence "The sign was too far away for me to read it," say upon using two heads for simplicity, if the $\mathrm{Q}=$ " $i t$ " the first head focuses more on the "sign" word and the second head focuses on "me" word. With the darker color to show more weight on attention, we have the final context representation will be focusing on those superior representations ("sign" word above " $m e "$ word above other words in the sentence) -more reading in [30], [29].

\section{EXPERIMENTAL SETUP}

We consider six translation tasks: three translations of nonconversational passages from English to Indonesian using Seq2Seq, and the remain three are from Indonesian to English, each using the RNN, ConvSeq2Seq, and MHA. The parallel datasets are from https: //github.com/desmond86/Indonesian-English-Bilingual-Corpus. As programming tools, we use PyTorch CUDA and Spacy (NLP).

\subsection{Dataset}

For English to Indonesian, we use three datasets: AusAid, Odb data, and Smeru datasets.

- First, the AusAid training set consists of 3112 manually aligned sentence pairs divided into four parts: 2489 sentences in the training set, 311 sentences in the testing set, 156 sentences in the validation set, and 156 sentences tuning set. The theme of AusAid contains policy news about Indonesia. The number of unique tokens in source vocab is 2771 tokens, while in target vocab consists of 2647 unique tokens.

- Second, the Odb data training set consists of 9823 manually aligned sentence pairs divided into four parts: 7858 sentences in the training set, 982 sentences in the testing set, 491 sentences in the validation set, and 492 sentences in tuning set. The theme of Odb data in religion news. The number of unique tokens in source vocab is 5729 tokens, while in target vocab consists of 5716 unique tokens.

- Third, the Smeru training set consists of 10394 manually aligned sentence pairs divided into four parts: 5000 sentences in the training set, 2697 sentences in the testing set, 1348 sentences in the validation set, and 1349 sentences in tuning set. The theme of Smeru is local government news. The number of unique tokens in source vocab is 4536 tokens, while in target vocab consists of 4210 unique tokens.

For Indonesian to English, we use the AusAid dataset. It consists of 3112 manually aligned sentence pairs divided into four parts: 2489 sentences in the training set, 467 sentences in the testing set, and 156 sentences in the validation set. The number of unique tokens in source vocab is 2647 tokens, while in target vocab consists of 2771 unique tokens. The preprocessing for each sentence is as follows: first, we tokenize text from sentence into tokens with accepted tokens occurrence minimum is two times and the number of tokens maximum is 100 tokens. Second, we set hyperparameters of the NMT.

\subsection{Metrics}

We measure the translation quality with BiLingual Evaluation Understudy (BLEU) scores, GLEU (Google-BLEU) scores, and Word Error Rate (WER) scores [21], [33], [24]. BLEU is good to measure how many words overlap given any translation compared to a reference translation, giving higher scores to sequential words. BLEU does not consider different types of errors (insertions, substitutions, synonyms, paraphrases, stems). Moreover, the purpose of BLEU is to be a corpus measure, so it has undesirable properties for single sentences. GLEU is simpler than BLEU but much closer to human judgments. For example, in GLEU, an error caused by an untranslated word or phrase is handled well by computes n-gram precisions over the reference, but GLEU assigns more weight to n-grams that correctly changed from the source. In WER, the minimum number of edit distances (insertion, deletions, or substitutions) must change the hypotheses sentence into the reference. The limitation is that there are no details on the nature of translation errors. There is no distinction between a substitution error and a deletion followed by an insertion error.

\subsection{Hyperparameters and Training Details}

3.3.1 English-Indonesian. For all datasets, we use Seq2Seq approach. We train each model with sentence lengths of up to 100 words in the training data. The dimension of word embeddings is 256 , and all hidden sizes are 512 . In training, the batch size is 128. We set encoder layers to 10 , and the decoder layer is 10 . The 
size of the encoder kernel is 3 , and the decoder kernel is 3 . The dropout encoder is set to 0.25 , while the decoder dropout is also the same, 0.25 . The number of epochs is 100 , and the clip is 0.1 . For AusAid dataset, number of trainable parameters is $34,384,983$, and 36,716,628 for Odb data, and 35,638,642 for Smeru dataset. We use Adam [14] with a learning rate $=0.001$ as our optimization algorithm and use cross-entropy loss to define and minimize the loss. For the AusAid dataset, the Test Loss is 3.743, 3.951 (Odb), and 3.957 (Smeru). The Test PPL is 42.231 for the AusAid dataset, 51.974 for Odb data, and 52.302 for the Smeru dataset.

3.3.2 Indonesian-English. We use the AusAid dataset for all three approaches: RNN, ConvSeq2Seq, and MHA approach. We train each model with sentence lengths of up to 100 words in the training data.

- RNN: In training, we set the batch size to 128 . The dimension of encoder embeddings is 256 , and the dimension of the decoder embeddings is also the same, 256. The dimension of Encoder hidden embeddings is 512, and the dimension of the Decoder hidden embeddings is also the same, 512 . The dropout encoder is set to 0.5 , while the decoder dropout is also the same, 0.5 . The number of epochs is 100 , and the clip parameter is 1 . The number of trainable parameters is $12,788,691$. We use Adam [14] with a learning rate $=0.001$ as our optimization algorithm and use cross-entropy loss to define and minimize the loss. The Test Loss is 5.363, and the Test PPL is 213.265 . The clipping value is usually between 0.5 and 10, depending on how harsh we want to clip large gradients.

- ConvSeq2Seq: The dimension of word embeddings is 256, and all hidden sizes are 512. In training, we set batch size $=128$. Encoder layers are 10, and the decoder layer is 10 . The size of the encoder kernel is 3 , and the decoder kernel is 3 . The dropout encoder $=0.25$, while the decoder dropout is also the same, 0.25 . The number of epochs is 100 , and the clip sets to 0.1 . The number of trainable parameters is $34,416,851$. We use Adam [14] with a learning rate $=0.001$ as our optimization algorithm and use cross-entropy loss to define and minimize the loss. The Test Loss is 3.565, and the Test PPL $=35.333$.

- MHA: The dimension of hidden sizes is 256. In training, the batch size is 128 . Encoder layers are 3, and the decoder layer is 3 . The number of encoder heads is 8 , and the number of decoder heads is also 8. The dimension of Encoder position-wise feedforward is 512 , and the dimension of Decoder position-wise feedforward is also the same, 512 . The dropout encoder is set to 0.1 , while the decoder dropout is also the same, 0.1 . The number of epochs is 100 , and the clip is 1 . The number of trainable parameters is $6,099,411$. We use Adam [14] with a learning rate $=0.0005$ as our optimization algorithm and use cross-entropy loss to define and minimize the loss. The Test Loss is 4.049, and the Test PPL is 57.355.

\section{RESULTS}

We first evaluate the metric results (BLEU, GLEU, WER) in Section 4.1, followed by discussion/future work in Section 4.2. For further inspection, the attention results are in Appendices.

\subsection{BLEU/GLEU/WER Results}

Table 1 shows, in general, for both English to Indonesian and Indonesian to English, the order from best to worst measured using both BLEU and GLEU are as follows: 1) ConvSeq2Seq, 2) Seq2Seq, 3) MHA, and 4) RNN. Results from the BLEU Sentence also show similar trends (measured using the same dataset, viz. AusAid). Table 1 shows a trend that BLEU Sentence (train) > BLEU Sentence (test) $>$ BLEU Sentence (valid).

While in the BLEU Sentence metric, the BLEU Sentence scores are computed by averaging BLEU scores of each sentence, in the BLEU Corpus metric, it is a different way. In BLEU Corpus, we sum the numerator and denominators for each pair of hypothesisreference(s) before the division. From Table 1, the order from best to worst measured using BLEU is as follows: 1) ConvSeq2Seq, 2) Seq2Seq, 3) RNN, and 4) MHA (MHA). RNN advantage is varied in length, whereas the Convolutional neural network has a fixed length. MHA allows the model to jointly attend to information from different representation subspaces at different positions.

$\mathrm{N}$-gram refers to $\mathrm{n}$ consecutive words in a sentence. From Table 1 , there are three metrics with grams: BLEU n-gram 1, BLEU n-gram, and BLEU n-gram geo. We can get BLEU-n scores using both sentence and corpus computations and the weights parameter with length $=4$, where every index contains a weight corresponding to its respective $n$-gram, are $[0.25,0.25,0.25,0.25]$. The computation of BLEU n-gram scores is by default (all n-grams are equal in a geometric mean). The BLEU n-gram 1 is an average of BLEU Sentence of hypothesis-references. The difference between the BLEU n-gram 1 with the BLEU n-gram is that the BLEU n-gram is an average of BLEU Sentence of hypothesis-references regarding weights. BLEU $\mathrm{n}$-gram geo is the geometric mean. Table 1 shows a trend that BLEU n-gram 1 (train) $\geq$ BLEU n-gram 1 (test) $\geq$ BLEU n-gram 1 (valid). A similar trend also for the BLEU n-gram and the BLEU geo. The brevity penalty penalizes sentences that are too short. To compute the BLEU score, we multiply the Brevity penalty with the Geometric average.

From Table 1, for the Seq2Seq approach for English to Indonesian, according to the BLEU results, the best setup is NMT with batch size 128 , embedding dimension 256, hidden dimension 512, encoder layers 10 , decoder layers 10 , encoder kernel size 3, decoder kernel size 3 , encoder dropout 0.25 , decoder dropout 0.25 , clip parameter 0.1 , and the number of epochs 100 .

GLEU is similar to BLEU but more like human judgment. Table 1 shows similar trend results for BLEU and GLEU (Google BLEU). The order from best to worst for macro view (BLEU Sentence or GLEU avg) is the same: 1) ConvSeq2Seq, 2) Seq2Seq, 3) MHA (MHA), and 4) RNN (RNN). Likewise, the order from best to worst for micro view (BLEU Corpus or GLEU Corpus) is the same: 1) ConvSeq2Seq, 2) Seq2Seq, 3) RNN, and 4) MHA. Figure 5 (left) shows that GLEU scores using Ausaid dataset, the ConvSeq2Seq (Ausaid5) outperform other methods. The trend is that GLEU on train set is better than GLEU on test set or validation set from phrase investigation. The GLEU scores decrease as a sentence becomes longer and longer. From Figure 5 (left), GLEU 1-gram to 2-gram (train) $\geq$ GLEU 1-gram to 3-gram (train) $\geq$ GLEU 1-gram to 4-gram (train). GLEU 1-gram to 2-gram (test) $\geq$ GLEU 1-gram to 3-gram (test) $\geq$ GLEU 1-gram 
Table 1: Evaluation of Results

\begin{tabular}{|c|c|c|c|c|c|c|}
\hline Metric & AusAid $^{1}$ & $\mathrm{Odb}^{2}$ & Smeru $^{3}$ & AusAid $^{4}$ & Ausaid $^{5}$ & AusAid $^{6}$ \\
\hline BLEU Sentence (train) & 38.99 & 31.46 & 34.39 & 37.17 & 42.59 & 40.13 \\
\hline BLEU Sentence (test) & 38.28 & 30.01 & 33.31 & 33.26 & 41.54 & 39.20 \\
\hline BLEU Sentence (valid) & 37.99 & 30.68 & 32.47 & 31.96 & 40.13 & 38.90 \\
\hline BLEU Corpus (train) & 40.80 & 41.41 & 43.23 & 36.55 & 42.91 & 36.41 \\
\hline BLEU Corpus (test) & 32.53 & 37.76 & 35.15 & 40.47 & 31.74 & 38.95 \\
\hline BLEU Corpus (valid) & 35.54 & 39.79 & 35.49 & 40.50 & 30.36 & 42.39 \\
\hline BLEU (n-gram 1 (train)) & 38.99 & 31.46 & 34.39 & 37.17 & 42.59 & 40.13 \\
\hline BLEU (n-gram 1 (test)) & 38.28 & 30.01 & 34.39 & 33.26 & 41.54 & 39.20 \\
\hline BLEU (n-gram 1 (valid)) & 37.99 & 30.68 & 74.01 & 31.96 & 40.13 & 38.90 \\
\hline BLEU (n-gram (train)) & 38.99 & 31.46 & 33.31 & 37.17 & 42.59 & 40.13 \\
\hline BLEU (n-gram (test)) & 38.28 & 30.01 & 33.31 & 33.26 & 41.54 & 39.20 \\
\hline BLEU (n-gram (valid)) & 37.99 & 30.68 & 74.01 & 31.96 & 40.13 & 38.90 \\
\hline BLEU (n-gram geo (train)) & 74.01 & 74.01 & 32.47 & 74.01 & 74.01 & 74.01 \\
\hline BLEU (n-gram geo (test)) & 74.01 & 74.01 & 32.47 & 74.01 & 74.01 & 74.01 \\
\hline BLEU (n-gram geo (valid)) & 74.01 & 74.01 & 74.01 & 74.01 & 74.01 & 74.01 \\
\hline GLEU avg (train) & 1.12 & 1.07 & 2.00 & 1.12 & 1.22 & 1.16 \\
\hline GLEU avg (test) & 1.10 & 0.99 & 1.94 & 0.95 & 1.16 & 1.10 \\
\hline GLEU avg (valid) & 1.14 & 1.10 & 2.15 & 0.95 & 1.28 & 1.24 \\
\hline GLEU corpus (train) & 37.17 & 33.23 & 39.48 & 23.41 & 41.05 & 15.19 \\
\hline GLEU corpus (test) & 20.40 & 18.00 & 20.21 & 9.30 & 19.19 & 11.81 \\
\hline GLEU corpus (valid) & 21.56 & 17.27 & 20.03 & 8.12 & 18.50 & 10.75 \\
\hline
\end{tabular}

1,2,3 using Seq2Seq (English-Indonesian), ${ }^{4}$ using RNN (Indonesian-English), ${ }^{5}$ using ConvSeq2Seq (Indonesian-English), ${ }^{6}$ using MHA (Indonesian-English).
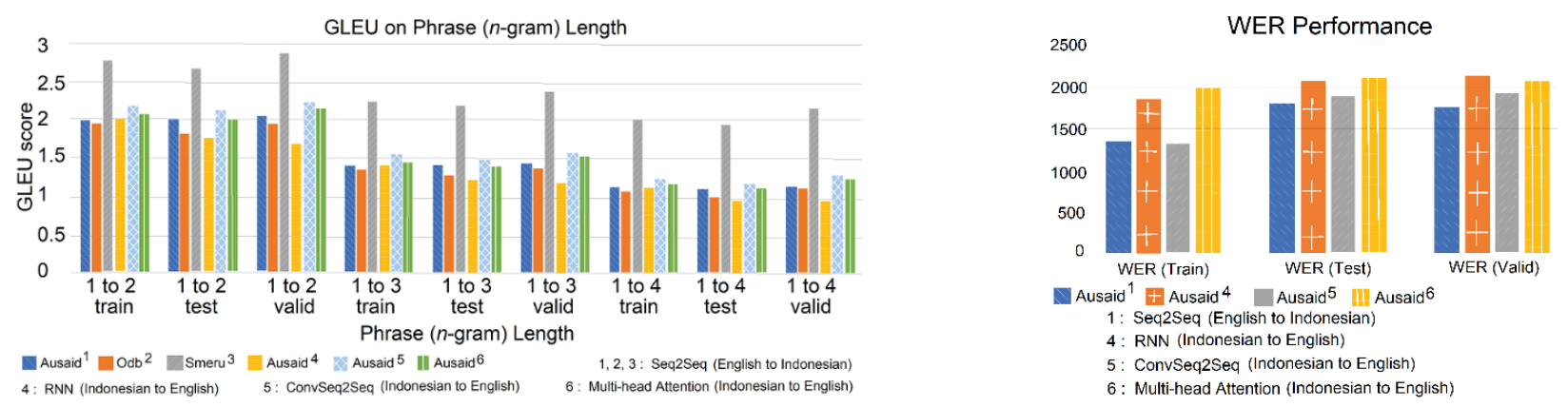

Figure 5: RNN with attention implemented in Indonesian-English. GLEU (left). WER (right).

to 4-gram (test). GLEU 1-gram to 2-gram (valid) $\geq$ GLEU 1-gram to 3-gram (valid) $\geq$ GLEU 1-gram to 4-gram (valid).

Any machine translation is looking for a system with a minimum Word Error Rate (WER). For example, from Figure 5 (right), Seq2Seq and ConvSeq2Seq have minimum WER, followed by RNN and MHA.

\subsection{Discussion/Future Work}

From Section 4.1, in general, for both English to Indonesian and Indonesian to English, the order from best to worst measured using both BLEU and GLEU are as follows: 1) ConvSeq2Seq, 2) Seq2Seq, 3) MHA (MHA), and 4) RNN (RNN). In addition, there are several tuning factors that we describe here as a discussion for future work to improve those NMT performances (see Section 4.2).
First, experiment with input formats. Morphemes are the smallest semantic unit. For neural machine translation, we can have different translation granularities: sentence-level [13], [32], [19], [35], word-level, character-level, hybrid (a combination of word and character-level), byte pair encoding (BPE), and Word-piece encoding. Nowadays, progress in more powerful chips leads to an increase in computing power. This situation, combined with the advance in the deep neural network, resulted in better quality performance of NMT. Systems such as Google Neural Machine Translation Systems (GNMT) [13] consider the entire input sentence as a unit translation. The advantage is that if a system translates the entire sentence without chunking it since it is complete, we can capture the semantic very well; thus, the predicted translation sentence sounds more natural. GNMT uses a variant of the word-piece and sentence-piece 


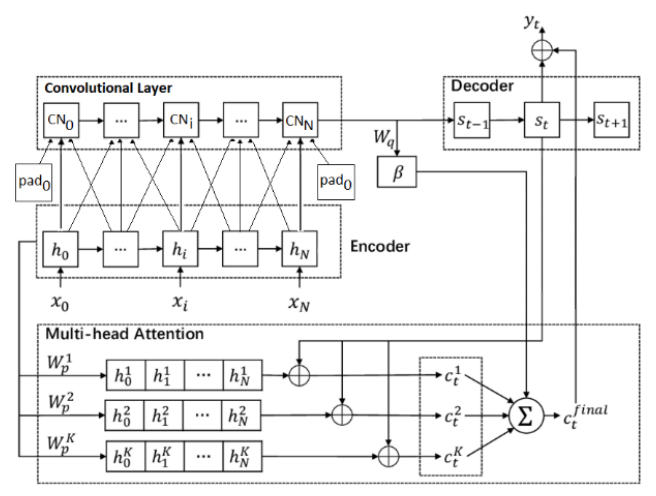

Figure 6: One-layer ConvSeq2Seq + MHA.

model. [17] show that a pure character-level Seq2Seq is better than a word-level model, except that the training time consumes much time (weeks, viz. third quarter to a month). While BPE is a kind of compression algorithm, where the most frequent byte pair is a new byte [22], the result from fully character-level of NMT without explicit segmentation [16] still perform better than BPE. Hybrid NMT translate mostly at the word level and only go to the character level when needed. From [17], the hybrid NMT shows more than 2 BLEU improvement scores over a copy mechanism to fill in rare words. For rare words and languages with lots of morphology, FastText embeddings represent words as a sum of char n-grams augmented with boundary symbols and as the whole word; it offers efficient word2vec by enriching word vectors with sub word information [4].

Second, explore the domain adaptation approach. The parallel corpus used in research can be translated manually by humans or automatically by the system. There is a term of "in" and "out" of the domain. A domain can be a speech-language, colloquial, formal written language, or any specific topic such as patent domain and scientific domain, or multi-domain (appending domain tag to the source to help NMT decoder to generate sentences for the specific domain). Then the tuning factor is adjusted. [6] shows that by training an NMT model on an out-of-domain parallel corpus, finetune the model on a parallel corpus with a mix of the in-domain and out-of-domain corpora (mixed fine-tuning) gives the best results. Such examples of variations are: without tags, fine-tuning on a particular defined domain, training on subset domains, are ideas to improve.

Third, improve the neural network model/architecture. We can do this by mixture models. Moreover, also we can explore multilayer/stacking, layer normalization, attention strategies are some examples of those strategies. As an example, ConvSeq2Seq with MHA is our proposed system to improve our best NMT system with attention (Figure 6). The figure remains an open discussion.

NMT such as RNNs can be stacked in layers, forming a grid [11]. We observed empirically that deep RNNs work better than shallower ones. In [28], it is reported that a four-layers deep architecture was crucial in achieving good machine- translation performance in an encoder-decoder framework. Also, from [12], it is reported that improvement showed by moving from a one-layer Bi-RNN to architecture with layers.
Normalization layers [1] in the model aim to speed up and stabilize the learning process. We can use batch normalization if training with large batches is not an issue and if the network does not have any recurrent connections. If the number of training batches is small, we can use group normalization with weight standardization. For training complex layers, instead of batch normalization, we can use group-of-normalization with Weight Standardization. The location of normalization layers is usually between the Linear/Conv/RNN layer and the ReLU non-linearity (hyperbolic tangent). Therefore, with the position, when the activations reach the Nonlinear activation function, the activations are equally centered around zero. The situation will avoid the dead neurons that never get activated due to wrong random initialization and improve the training process.

Fourth, multilayer decoding, layer normalization, and regularization techniques such as dropout [25], [9] and tune out [3] also helpful for NMT fine-tuning.

\section{CONCLUSION}

This study discusses the essentials of neural machine translation techniques (Seq2Seq, ConvSeq2Seq, RNN, and MHA while implemented in formal passages in English-Indonesian and IndonesianEnglish. The order from best to worst measured using both BLEU and GLEU are as follows: 1) ConvSeq2Seq, 2) Seq2Seq, 3) MHA, and 4) RNN.

The best method, the ConvSeq2Seq, is an improvement process of the Seq2Seq model. As in other NMT, the use of attention helps improve the NMT system. The rationale for the good performance of ConvSeq2Seq with attention is as follows. The ConvSeq2Seq uses convolutions instead of regular RNNs. Convolutions allow overcoming highly sequential processing inherent with RNN. The purpose of stacked convolutions in ConvSeq2Seq with the attention approach is to deal with long input sequences. It is because ConvSeq2Seq has a fixed size. Consequently, the stacking increases model parameters and the length between tokens because the receptive field grows faster to model long-range dependencies between different words. The convolution blocks themselves also use residual connections to allow for better gradient flow. Finally, the ConvSeq2Seq with the attention uses GLUs (Gated Linear Units) to non-linearity large scale problems. The ConvSeq2Seq with the attention approach does not depend on the previous time step's computations and it allows parallelization over each element in a sequence. Contrary to the situation, in RNNs, we must maintain a hidden state of the entire past that prevents parallel computation within a sequence.

In some cases, for testing and valid dataset of AusAid data, RNN and MHA show better performance than ConvSeq2Seq. MHA can perform more flexible transformations than single-head attention because MHA computes the scaled dot-product attention multiple times in parallel, rather than only computing the attention once. $\mathrm{RNN}$ has the advantage that it can process inputs of any length, and the model is to remember every information along the time which is very helpful in any time series predictor. Also, even if the input size is large, the model size does not increase. Another advantage is that the weights in RNN share during the time steps. $\mathrm{RNN}$ can use its internal memory for processing the arbitrary series of inputs which is not the case with feedforward neural networks. 
However, the RNN also has drawbacks as follows. First, because of its recurrent nature, the computation is slow. Also, the training of RNN models can be difficult. If we use ReLU or Tanh as activation functions, it becomes challenging to process sequences that are very long.

From the Discussion Section, we have four essential factors to explore further to improve an NMT system: experiment with input formats (sentence-level, word-level, character-level, hybrid (a combination of word and character-level, BPE, and Word-piece encoding), explore the domain adaptation approach, improve the neural network model/architecture (combine network architectures, multilayer/stacking, layer normalization, attention strategies), and do multilayer decoding, layer normalization, and regularization techniques (dropout, tune out).

\section{REFERENCES}

[1] Jimmy Lei Ba, Jamie Ryan Kiros, and Geoffrey E. Hinton. 2016. Layer Normalization. arxiv:1607.06450. Retrieved from https://arxiv.org/abs/1607.06450v1

[2] Dzmitry Bahdanau, Kyunghyun Cho, and Yoshua Bengio. 2014. Neural Machine Translation by Jointly Learning to Align and Translate. arXiv:1409.0473. Retrieved from http://arxiv.org/abs/1409.0473

[3] Antonio Valerio Miceli Barone, Barry Haddow, Ulrich Germann, and Rico Sennrich. 2017. Regularization techniques for fine-tuning in neural machine translation. In Proceedings of the 2017 Conf. on EMNLP, Sep., 2017, Copenhagen, Denmark. ACL, 1489-1494. https://doi.org/10.18653/v1/D17-1156

[4] Piotr Bojanowski, Edouard Grave, Armand Joulin, and Tomas Mikolov. 2017. En riching Word Vectors with Subword Information. Transactions of the Association for Computational Linguistics 5 (2017), 135-146. DOI: https://doi.org/10.1162/ tacl_a_00051

[5] Kyunghyun Cho, Bart van Merriënboer, Caglar Gulcehre, Dzmitry Bahdanau, Fethi Bougares, Holger Schwenk, and Yoshua Bengio. 2014. Learning Phrase Representations using RNN Encoder-Decoder for Stat. Mach. Translation. In Proc. 2014 EMNLP, October 2014, Doha, Qatar. ACL, 1724-1734. https://aclanthology. org/D14-1179

[6] Chenhui Chu, Raj Dabre, and Sadao Kurohashi. 2017. An Empirical Comparison of Domain Adaptation Methods for Neural Machine Translation. In Proc. 55th Annual Meeting of ACL (Vol. 2: Short Papers), July 2017, Vancouver, Canada. ACL, 385-391. https://doi.org/10.18653/v1/P17-2061

[7] Chenhui Chu and Rui Wang. 2018. A Survey of Domain Adaptation for Neural Machine Translation. In Proceedings of the 27th International Conference on Computational Linguistics, Aug, 2018, SantaFe, New Mexico, USA. ACL, 13041319. https://aclanthology.org/C18-1111

[8] Raj Dabre, Chenhui Chu and Anoop Kunchukuttan. 2020. A Survey of Multilingual Neural Machine Translation. J. ACM Computing Surveys 53, 5, Article 99 (Oct. 2020), 38 pages. DOI: https://doi.org/10.1145/3406095

[9] Yarin Gal and Zoubin Ghahramani. 2016. A Theoretically Grounded Application of Dropout in Recurrent Neural Networks. In Proceedings of the 30th International Conference on Neural Information Processing Systems (NIPS '16), December, 2016. ACM Inc., New York, NY, 1027-1035. https://dl.acm.org/doi/10.5555/ 3157096.3157211

[10] Jonas Gehring, Michael Auli, David Grangier, Denis Yarats, and Yann N. Dauphin. 2017. Convolutional Sequence to Sequence Learning. In Proceedings of the 34th International Conference on Machine Learning (ICML '17), August 2017. ACM Inc., New York, NY, 1243-1252. https://dl.acm.org/doi/10.5555/3305381.3305510

[11] Salah El Hihi and Yoshua Bengio. 1996. Hierarchical Recurrent Neural Networks for Long-Term Dependencies. In NIPS'95: Proceedings of the 8th International Conference on Neural Information Processing Systems, November, 1995. MIT Press,Cambridge, MA, 493-499. https://dl.acm.org/doi/10.5555/2998828.2998898

[12] Ozan İrsoy, Claire Cardie. 2014. Opinion Mining with Deep Recurrent Neural Networks. In Proceedings of the 2014 Conference on Empirical Methods in Natural Language Processing(EMNLP), October 2014, Doha, Qatar. ACL, 720-728. https://doi.org/10.3115/v1/D14-1080

[13] Melvin Johnson, Mike Schuster, Quoc V. Le, Maxim Krikun, Yonghui Wu, Zhifeng Chen, Nikhil Thorat, Fernanda Viegas, Martin Wattenberg, Greg Corrado, Macduff Hughes, and Jeffrey Dean. 2017. Google's Multilingual Neural Mach. Translation System: Enabling Zero-Shot Translation. Transactions of the Association for Computational Linguistics 5 (2017), 339-351. DOI: https: //doi.org/10.1162/tacl_a_00065

[14] Diederik P. Kingma and Jimmy Ba. 2017. Adam: A Method for Stochastic Optimization. arXiv:1412.6980. Retrieved from https://arxiv.org/abs/1412.6980

[15] Yann LeCun and Yoshua Bengio. 1998. Convolutional networks for images, speech, and time series. In The handbook of brain theory and neural networks, October
1998. MIT Press, Cambridge, MA, 255-258. https://dl.acm.org/doi/10.5555/303568. 303704

[16] Jason Lee, Kyunghyun Cho, Thomas Hofmann. 2017. Fully Character-Level Neural Machine Translation without Explicit Segmentation. Transactions of the Association for Computational Linguistics 5 (2017), 365-378. DOI: https: //doi.org/10.1162/tacl_a_00067

[17] Minh-Thang Luong and Christopher D Manning. 2016. Achieving open vocabulary neural machine translation with hybrid word-character models. In Proc. of the 54th Annual Meeting of the Association for Computational Linguistics (Volume 1: Long Papers), Aug, 2016, Berlin, Germany. ACL, 1054-1063. https://doi.org/10.18653/v1/P16-1100

[18] Thang Luong, Hieu Pham, and Christopher D. Manning. 2015. Effective Approaches to Attention-based Neural Machine Translation. In Proceedings of the 2015 Conf. on EMNLP, Sep, 2015, Lisbon, Portugal. ACL, 1412-1421. https: //doi.org/10.18653/v1/D15-1166

[19] Elman Mansimov, Gabor Melis, and Lei Yu. 2020. Capturing document context inside sentence-level neural machine translation models with self-training. arXiv: 2003.05259. Retrieved from https://arxiv.org/abs/2003.05259

[20] Graham Neubig. 2017. Neural Machine Translation and Sequence-to-sequence Models: A Tutorial. arXiv:1703.01619. Retrieved from https://arxiv.org/abs/1703. 01619

[21] Kishore Papineni, Salim Roukos, Todd Ward, and Wei-Jing Zhu. Bleu: a method for automatic evaluation of machine translation. In Proceedings of the 40th Annual Meeting of the Association for Computational Linguistics, July, 2002, Philadelphia, Pennsylvania. ACL, 311-318. https://doi.org/10.3115/1073083.1073135

[22] Rico Sennrich, Barry Haddow, and Alexandra Birch. 2016. Improving Neural Machine Translation Models with Monolingual Data. In Proceedings of the 54th Annual Meeting of the Association for Computational Linguistics (Volume 1: Long Papers), Aug 2016, Berlin, Germany. ACL, 1715-1725. https://doi.org/10. 18653/v1/P16-1009

[23] Rico Sennrich, Barry Haddow, and Alexandra Birch. 2016b. Edinburgh Neural Machine Translation Systems for WMT 16. In Proc. of the First Conference on Machine Translation: Volume 2, Shared Task Papers, Aug, 2016, Berlin, Germany. ACL, 371-376. https://doi.org/10.18653/v1/W16-2323

[24] Xingyi Song, Lucia Specia, and Trevor Cohn. 2013. BLEU deconstructed: Designing a Better MT Evaluation Metric. International Journal of Computational Linguistics and Applications 4, 2 (Jul-Dec 2013), 29-44. DOI: http://citeseerx.ist. psu.edu/viewdoc/summary?doi=10.1.1.365.5678

[25] Nitish Srivastava, Geoffrey E Hinton, Alex Krizhevsky, Ilya Sutskever, and Ruslan Salakhutdinov. 2014. Dropout: a simple way to prevent neural networks from overfitting. Journal of Machine Learning Research 15, 1 (Jan. 2014), 1929-1958. DOI: https://dl.acm.org/doi/10.5555/2627435.2670313

[26] Felix Stahlberg. 2020. Neural Machine Translation: A Review and Survey. arXiv: 1912.02047. Retrieved from https://arxiv.org/abs/1912.02047

[27] Ralf C. Staudemeyer and Eric Rothstein Morris. 2019. Understanding \{LSTM\} - a tutorial into Long Short-Term Memory Recurrent Neural Networks. arXiv: 1909.09586. Retrieved from http://arxiv.org/abs/1909.09586

[28] Ilya Sutskever, Oriol Vinyals, and Quoc V. Le. 2014. Sequence to Sequence Learning with Neural Networks. In Proceedings of the 27th International Conference on Neural Information Processing Systems (NIPS '14) - Volume 2, December, 2014. MIT Press, Cambridge, MA, 3104-3112. https://dl.acm.org/doi/10.5555/2969033. 2969173

[29] Chonghyang Tao, Shen Gao, Mingyue Shang, Wei Wu, Dongyan Zhao, and Rui Yan. 2018. Get The Point of My Utterance! Learning Towards Effective Responses with MHA Mechanism. In Proc. 27th Int. Joint Conference on Artificial Intelligence, July, 2018. IJCAI, 4418-4424. https://doi.org/10.24963/ijcai.2018/614

[30] Ashish Vaswani, Noam Shazeer, Niki Parmar, Jakob Uszkoreit, Llion Jones, Aidan N. Gomez, Lukasz Kaiser, and Illia Polosukhin. 2017. Attention Is All You Need. In Proceedings of the 31st Int. Conf. on NIPS (NIPS '17), December, 2017. Curran Associates Inc., Red Hook, NY, 6000-6010. https://dl.acm.org/doi/10.5555/3295222. 3295349

[31] Alex Waibel, Toshiyuki Hanazawa, Geoffrey Hinton, Kiyohiro Shikano, and Kevin J. Lang. 1989. Phoneme Recognition using Time-delay Neural Networks. IEEE transactions on acoustics, speech, and signal processing 37, 3 (March 1989), 328-339. DOI: https://doi.org/10.1109/29.2170131

[32] Yining Wang, Long Zhou, Jiajun Zhang, and Chengqing Zong. 2017. Word, Subword or Character? An Empirical Study of Granularity in Chinese-English NMT. arXiv:1711.04457. Retrieved from https://arxiv.org/abs/1711.04457

[33] Yonghui Wu, Mike Schuster, Zhifeng Chen, Ouoc V. Le, Mohammad Norouzi, Wolfgang Macherey, Maxim Krikun, Yuan Cao, Qin Gao, Klaus Macherey, Jeff Klingner, Apurva Shah, Melvin Johnson, Xiaobing Liu, Łukasz Kaiser, Stephan Gouws, Yoshikiyo Kato, Taku Kudo, Hideto Kazawa, Keith Stevens, George Kurian, Nishant Patil, Wei Wang, Cliff Young, Jason Smith, Jason Riesa, Alex Rudnick, Oriol Vinyals, Greg Corrado, Macduff Hughes, and Jeffrey Dean. 2016. Google's Neural Machine Translation System: Bridging the Gap between Human and Machine Translation. arXiv:1609.08144. Retrieved from https://arxiv.org/abs/ 1609.08144 
Table 2: Attention Results

\begin{tabular}{|c|c|}
\hline Dataset & Sentence \\
\hline Ausaid ${ }^{1}$ & src = ['providing', 'technical', 'guidance', 'and', 'evaluation', 'on', 'improvements', 'to', 'the', 'legal', 'system', ';'] \\
\hline Ausaid $^{1}$ & $\operatorname{trg}=$ ['memberikan', 'bantuan', 'teknis', 'dan', 'evaluasi', 'terhadap', 'peningkatan', 'di', 'sistem', 'hukum', ';'] \\
\hline Ausaid $^{1}$ & predicted trg = ['memberikan', 'panduan', 'teknis', 'dan', 'evaluasi', 'pada', 'sistem', 'hukum', ';', '<eos>'] (Good) \\
\hline $\mathrm{Odb}^{2}$ & src = ['we', "'re", 'wrong', ',, 'of', 'course'] \\
\hline $\mathrm{Odb}^{2}$ & $\operatorname{trg}=$ ['sikap', 'kita', 'ini', 'tentu', 'salah'] \\
\hline $\mathrm{Odb}^{2}$ & predicted trg = ['kita', 'terkejut', ',, 'kita', 'tidak', 'pernah', 'beres', '<eos >'] (Bad) \\
\hline Smeru ${ }^{3}$ & $\begin{array}{l}\text { src = ['consequently', ',, 'the', 'local', 'government', 'needs', 'to', 'carry', 'out', 'an', 'in', '-', 'depth', 'study', 'before', } \\
\text { 'imposing', 'these', 'charges'] }\end{array}$ \\
\hline Smeru $^{3}$ & $\begin{array}{l}\text { trg = ['oleh', 'karena', 'itu', ', ', 'pemda', 'perlu', 'melakukan', 'kajian', 'mendalam', 'sebelum', 'memberlakukan', 'pungutan', } \\
\text { 'tersebut'] }\end{array}$ \\
\hline $\mathrm{Smeru}^{3}$ & $\begin{array}{l}\text { predicted trg = ['akibatnya,, ', 'pemda', 'perlu', 'disesuaikan', 'untuk', 'melakukan', 'kombinasi', 'dan', '<unk>', '-', 'studi', } \\
\text { 'ini', 'sebelum', 'pungutan', 'pungutan', '<eos>'] (Good) }\end{array}$ \\
\hline Ausaid $^{4}$ & $\begin{array}{l}\text { src = ['pendekatan', 'aipj', 'dalam', 'bekerja', 'di', 'bidang-bidang,, 'yang', 'telah', 'diidentifikasi', 'akan', 'mencakup', } \\
\text { 'komitmen', 'kuat', 'untuk', 'mengembangkan', 'kemitraan', 'dan', 'mempromosikan', 'dialog', 'kebijakan', 'antara', } \\
\text { 'lembaga-lembaga', 'hukum', 'dan', 'keadilan', 'indonesia', 'dan', 'australia'] }\end{array}$ \\
\hline Ausaid $^{4}$ & $\begin{array}{l}\operatorname{trg}=\text { ['aipj', 's', 'approach', 'to', 'working', 'in', 'the', 'areas', 'identified', 'will', 'involve', 'a, 'strong', 'commitment', 'to', } \\
\text { 'developing', 'partnerships', 'and', 'promoting,', 'policy', 'dialogue', 'between', 'indonesian', 'and', 'australian', 'law', 'and', } \\
\text { 'justice', 'institutions'] }\end{array}$ \\
\hline Ausaid $^{4}$ & $\begin{array}{l}\text { predicted trg = ['aipj', 's', 'approach', 'to', 'the', 'partner', 'and', 'the', 'that', 'will', 'be', 'a', 'key', 'government', 'and', 'the', } \\
\text { 'indonesian', 'government', 'and', 'justice', 'institutions', 'and', 'justice', 'institutions', 'and', 'justice', 'institutions', 'and', } \\
\text { 'justice', 'institutions', 'and', 'justice', 'institutions', '<eos>'] (Bad) }\end{array}$ \\
\hline Ausaid $^{5}$ & $\begin{array}{l}\text { src = ['selain', 'itu', 'juga', 'secara', 'umum', 'terdapat', 'ketidakpercaya', 'terhadap', 'kapasitas', 'lembaga-lembaga', } \\
\text { 'hukum', 'dan', 'keadilan', 'negara', 'untuk', 'memberikan', 'layanan', 'hukum,, 'yang', 'berkualitas'] }\end{array}$ \\
\hline Ausaid $^{5}$ & $\begin{array}{l}\operatorname{trg}=\text { ['there', 'is', 'also', 'general', 'lack', 'of', 'faith', 'in', 'capacity', 'of', 'the', 'country', "s', 'law', 'and', 'justice', } \\
\text { 'institutions', 'to', 'deliver', 'quality', 'legal', 'service'] }\end{array}$ \\
\hline Ausaid $^{5}$ & $\begin{array}{l}\text { predicted trg = ['in', 'addition', 'to', 'the', 'importance', 'of', 'law', 'and', 'justice', 'institutions', 'reform', 'to', 'provide', } \\
\text { 'legal', 'services', '<eos>'] (Good) }\end{array}$ \\
\hline Ausaid $^{6}$ & $\begin{array}{l}\text { src = ['istilah', ', 'layanan', 'hukum', "', 'akan', 'digunakan', 'untuk', 'merujuk', 'ke', 'berbagai', 'layanan', 'yang', } \\
\text { 'disediakan', 'oleh', 'lembaga-lembaga,', 'sektor', 'hukum,, 'dan', 'keadilan', 'bagi', 'para', 'pencari', 'keadilan', 'dan', } \\
\text { 'masyarakat', 'pada', 'umumnya'] }\end{array}$ \\
\hline Ausaid $^{6}$ & $\begin{array}{l}\operatorname{trg}=\text { ['the', 'term', '“, 'legal', 'services', '”, 'will', 'be', 'used', 'to', 'refer', 'to', 'the', 'broad', 'range', 'of', 'services', 'provided', } \\
\text { 'by', 'law', 'and', 'justice', 'sector', 'institutions', 'to', 'justice', '-', 'seekers', 'and', 'the', 'community', 'at', 'large'] }\end{array}$ \\
\hline Ausaid $^{6}$ & $\begin{array}{l}\text { predicted trg = ['the', 'law', 'and', 'justice', 'sector', 'will', 'be', 'conducted', 'to', 'be', 'conducted', 'at', 'the', 'services', 'and', } \\
\text { 'the', '<unk>', 'of', 'the', 'community', 's', 'law', 'and', 'justice', 'sector', '<eos>'] (Bad) }\end{array}$ \\
\hline
\end{tabular}

[34] Shuoheng Yang, Yuxin Wang, and Xiaowen Chu. 2020. A Survey of Deep Learning Techniques for Neural Machine Translation. arXiv: 2002.07526. Retrieved from https://arxiv.org/abs/2002.07526

[35] Pei Zhang, Xu Zhang, Wei Chen, Jian Yu, Yanfeng Wang, and Deyi Xiong. 2020 Learning Contextualized Sentence Representations for Document-Level Neural Machine Translation. arXiv:2003.13205. Retrieved from https://arxiv.org/abs/2003. 13205

[36] Jie Zhou, Ying Cao, Xuguang Wang, Peng Li, and Wei Xu. 2016. Deep Recurrent Models with Fast-Forward Connections for Neural Machine Translation. Transactions of the Association for Computational Linguistics 4 (2016), 371-383. DOI: https://doi.org/10.1162/tacl_a_00105

\section{A A APPENDICES}

Figure 7 randomly display the attention weights for different attention heads. Each horizontal axis represents the input query, while, for every attention head, the vertical axis is the output translation. The lighter squares symbolize larger weights, with the corresponding cases are in Table 2. The successful translation indicates by a diagonal down stair-ward from left to right trend in the translation matrix.

\section{A.1 Attention Results}




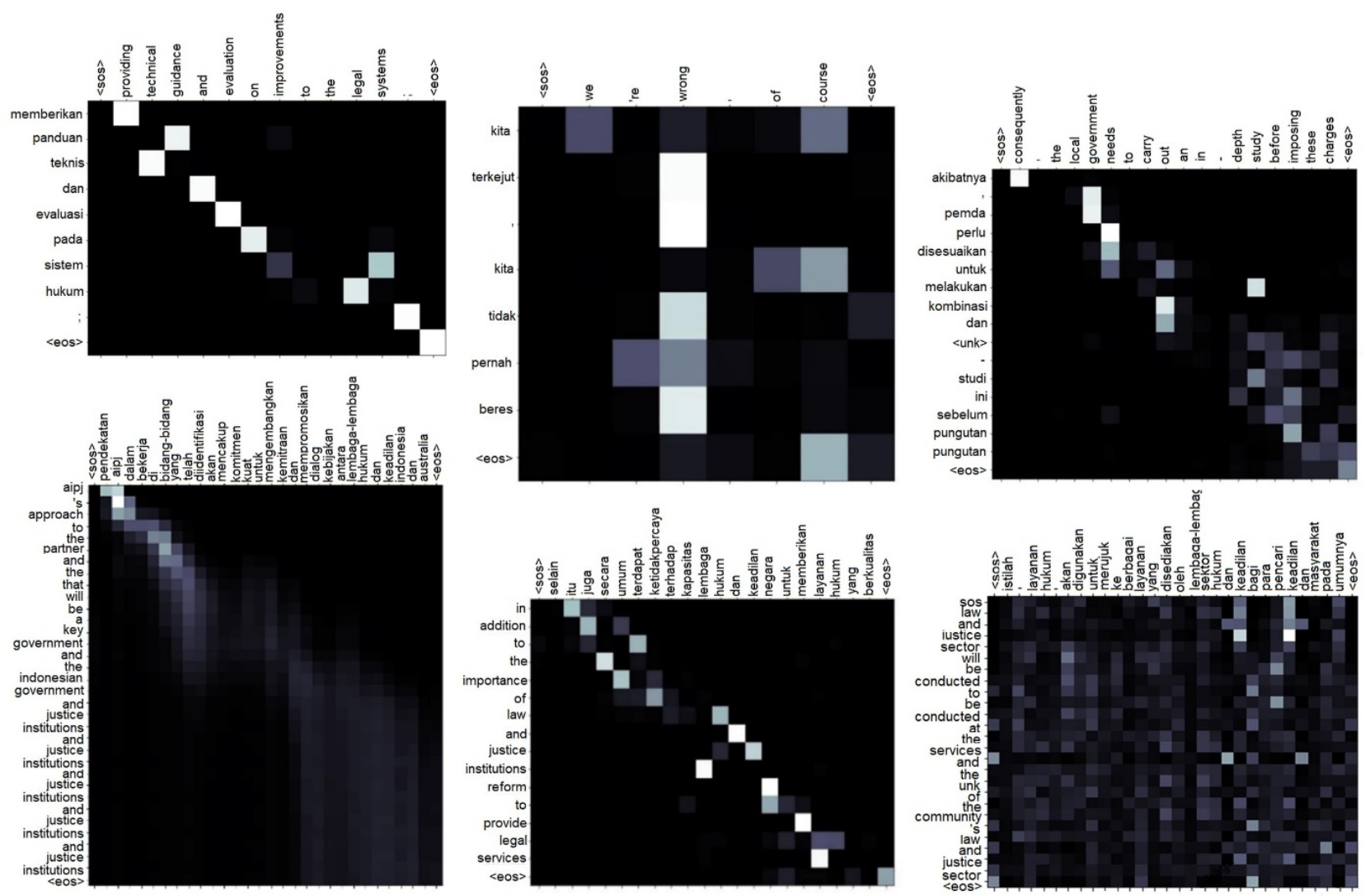

Figure 7: The corresponding visualization of attention results in Table 2. Seq2Seq (English-Indonesian) results on AusAid ${ }^{1}$ (top, left). Seq2Seq (English-Indonesian) results on $\mathrm{Odb}^{2}$ (top, center). Seq2Seq (English-Indonesian) results on Smeru ${ }^{3}$ (top, right). RNN (Indonesian-English) results on AusAid ${ }^{4}$ (bottom, left). ConvSeq2Seq (Indonesian-English) results on AusAid ${ }^{5}$ (bottom, center). MHA (Indonesian-English) results on AusAid ${ }^{6}$ (bottom, right). 